\title{
Pernicious anaemia presenting as catatonia without signs of anaemia or macrocytosis
}

\author{
S. Jauhar, A. Blackett, P. Srireddy and P. J. McKenna
}

\section{Summary}

Pernicious anaemia can present with psychiatric symptoms before haematological or neurological manifestations appear. We describe a young woman who presented with insidious onset catatonia without evidence of psychosis or depression. Blood count and mean cell volume were normal and neurological findings were equivocal. Low $B_{12}$ levels and intrinsic factor antibodies were found only by chance when they were included in a battery of further investigations. $B_{12}$ replacement was followed by prompt improvement. This case provides an argument for wider screening for $B_{12}$ deficiency in certain individuals with psychiatric disorders.

\section{Declaration of interest}

None.
Psychiatric disturbance was alluded to in Addison's original description of pernicious anaemia, and nowadays the disorder is recognised as being associated with depression, mania, psychosis and dementia. ${ }^{1}$ It is also known that psychiatric symptoms can precede the onset of anaemia or neurological features and can sometimes be seen in the absence of macrocytosis. ${ }^{2}$ A case report has recently added catatonia to the list of psychiatric syndromes associated with $\mathrm{B}_{12}$ deficiency. ${ }^{2}$ Here we present a further case, where catatonia was the presenting - and only - psychiatric feature of what turned out to be autoimmune $\mathrm{B}_{12}$ deficiency. Haematological abnormality was absent and neurological abnormality was subtle to the point of being easy to dismiss.

\section{Method}

A 27-year-old single croupier and singer of mixed race was compulsorily admitted after she was found to be living in a flat with no heating and electricity. She had stopped working around a year previously and had become withdrawn. There was no previous medical or psychiatric history. In hospital she was noted to be unkempt and vague; she would often stare blankly into space, had no spontaneous interaction with others, and at times was doubly incontinent. However, no affective or psychotic symptoms were elicited. She was treated with olanzapine with slight improvement. She was discharged by a mental health tribunal and then became lost to follow-up.

She was readmitted 6 months later, again in a state of selfneglect. She was very slow to initiate movements, took a long time to get out of bed, and would stand in the same position for long periods in the ward, often with her arms folded across her chest. She also followed members of staff around the ward repeating what they said. During an interview, she spent the whole time sitting in an erect posture in her chair with her hands clasped in a praying position. Her speech was reduced to greetings and giving answers to questions. She showed echolalia. Her affect was impassive and any changes of facial expression were few and slow. As previously, no psychotic or depressive symptoms could be elicited.

Neurological examination revealed a left palmomental reflex and a right upgoing plantar. She also had a positive Luria's (fist-edge-palm) test. Cognitively, she scored $67 / 100$ on the Addenbrooke's Cognitive Examination-Revised (ACE-R $)^{3}$ (normal > 82). Digit span was 4 forwards and 4 backwards. She showed borderline poor performance on story recall $(6 / 23$ idea units on the 'Brian Kelly' story from the Rivermead Behavioural Memory Test, ${ }^{4}$ normal range $\geqslant 6$ ) and poor verbal fluency (five words beginning with $\mathrm{P}$ in $1 \mathrm{~min}$ ). She was in the impaired range on two executive tests: the Hayling Test, ${ }^{5}$ which measures ability to inhibit prepotent responses (error score 43, scaled score 1), and the Brixton Test, ${ }^{5}$ a rule-shifting test conceptually similar to the Wisconsin Card Sorting Test (error score 39, scaled score 1).

Computed tomography (CT) scan was reported as showing mild frontal atrophy, but according to further neuroradiological opinions this was not unequivocally outside the normal range (Fig. 1). Magnetic resonance imaging was reported as showing only increased cerebrospinal fluid spaces around the hemispheres and increased third ventricle size. Single photon emission tomography scan was normal. Electroencephalogram (EEG) showed only bilateral posterior theta wave activity.

Because of the inconclusiveness of these findings, one of the authors (S.J.) undertook a raft of further investigations, including autoantibody screen, thyroid function tests, syphilis serology, serum $\mathrm{B}_{12}$ and folate, and voltage-gated potassium channel antibodies. The only abnormal finding was a low vitamin $\mathrm{B}_{12}$ of $159 \mathrm{ng} / \mathrm{l}$ (normal range 189-883 ng/l). Around this time her haemoglobin was $12.7-13.1 \mathrm{~g} / \mathrm{dl}$ on different occasions, with mean cell volume (MCV) of 86-94 fl (normal range 86-98 fl). Subsequently, antibodies to intrinsic factor were found, and the diagnosis of pernicious anaemia was made.

\section{Results}

Olanzapine, which had been recommenced shortly after admission (in addition to diazepam), did not result in obvious improvement over 4 weeks. However, less than 10 days after starting on hydroxycobalamine (intramuscularly, at a dosage of $1 \mathrm{mg}$ on alternate days for 10 days), nursing staff commented on a marked improvement in her behaviour and self-care. Her Addenbrooke's score (on parallel versions of the ACE-R) was 80/100 2 weeks after commencement of treatment and 90/100 4 weeks later. Digit span after 10 weeks of treatment was 6 forwards and 5 backwards and she had improved into the 'moderate average' range on the Brixton test (19 errors, scaled score 5); however, she remained in the impaired range on the Hayling Test (33 errors, scaled score 1). As she began to talk more, she described having had pins and needles in her hands and feet for approximately a year, which could have reflected spinal cord demyelination. No abnormalities were found on repeat neurological examination. A repeat CT scan 4 months after institution of $B_{12}$ replacement continued to show much the same degree of frontal sulcal widening, but was reported as normal.

She discontinued olanzapine 2 months after discharge from hospital. Four months later, after a 2-week delay in administration of her hydroxycobalamine injection, she had a brief, 2-week 


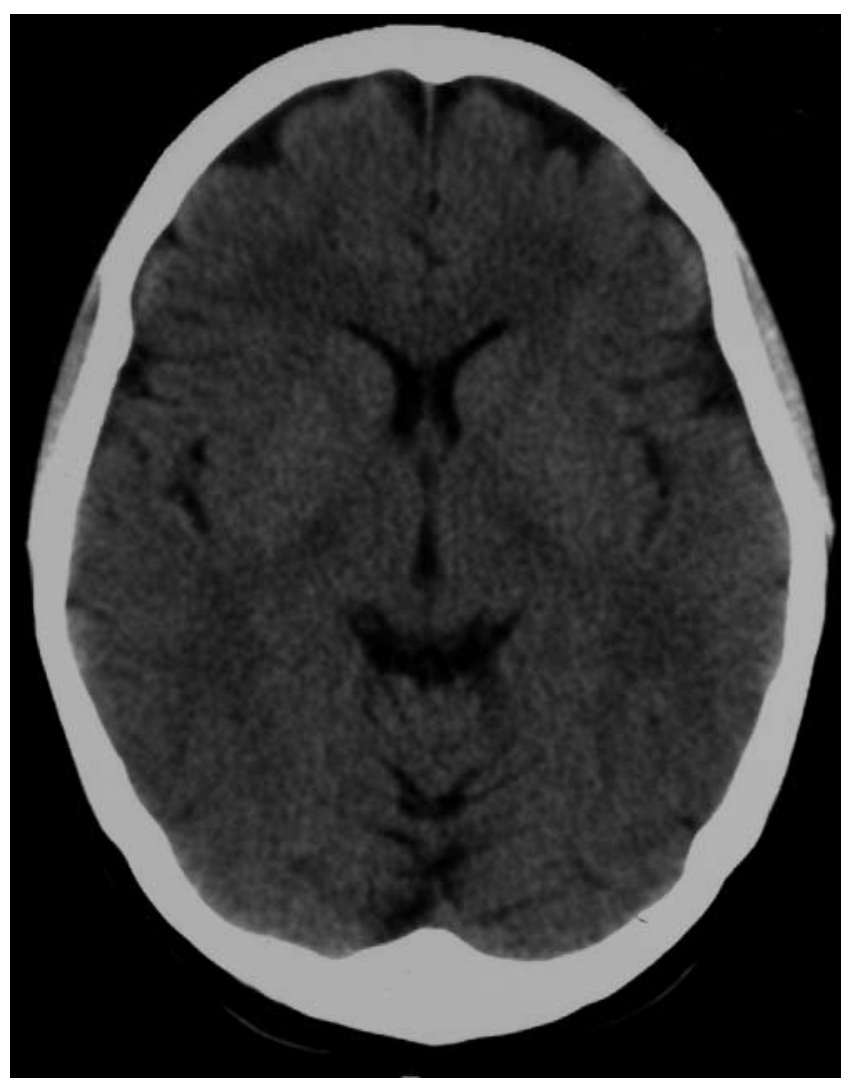

Fig. 1 Computed tomography scan.

period of catatonia, which resolved promptly to re-initiation of olanzapine. Currently, she is continuing to receive olanzapine and vitamin $\mathrm{B}_{12}$ injections, is out of hospital, lives independently in her own flat and is planning to enrol in a music course.

\section{Discussion}

This individual presented with marked social decline, self-neglect and later developed full-blown catatonic symptoms without evidence of psychotic or affective symptoms. Her neurological abnormalities were minimal, and whether there was any abnormality on brain imaging became the subject of debate among the clinicians and neuroradiologists involved in her case. Olanzapine brought about no improvement over 4 weeks but after pernicious anaemia was diagnosed hydroxycobalamine treatment was followed by rapid recovery.

A complicating factor was her relapse after discontinuing olanzapine and recovery after this drug was re-introduced. Two findings, however, argue against her having an independent diagnosis of schizophrenia. First, she did not experienced positive psychotic symptoms for more than 2 years of illness. Second, she originally showed a substantial degree of cognitive impairment, which then improved. This is inconsistent with schizophrenic cognitive impairment, which is now generally accepted as being largely unresponsive to treatment. ${ }^{6}$ On the other hand, vitamin $\mathrm{B}_{12}$ deficiency can cause cognitive impairment up to and including dementia; ${ }^{1}$ the degree to which this improves with $B_{12}$ replacement is variable, with 1 year having been suggested as a watershed for reversibility. ${ }^{7}$ Brain changes associated with $\mathrm{B}_{12}$ deficiency are less well documented, with different studies finding no abnormality, cerebral atrophy or leukoencephalopathy, again with varying degrees of reversibility. ${ }^{8}$
This person's lack of anaemia and her (at most) subtle neurological signs meant that vitamin $\mathrm{B}_{12}$ deficiency was not originally screened for. In 1988, Lindenbaum et al questioned the existing orthodoxy that $\mathrm{B}_{12}$ deficiency only presented with neurological disorder when anaemia was also present. ${ }^{9}$ They reviewed 141 people who were given a final diagnosis of clinical $\mathrm{B}_{12}$ deficiency-related neurological symptoms and found that 40 $(28 \%)$ had either no anaemia or no macrocytosis at initial presentation, and that in $19(13 \%)$ both haematological indices were normal. Six further individuals had anaemia, but with an MCV that was normal or low (in four of these there were independent causes for this). In some of these people diagnosis was delayed for several months or years.

This woman's case adds to the literature documenting the occurrence of catatonia in a wide range of systemic diseases, and supports the view that it is an independent psychiatric entity, not one that develops only in the context of a schizophrenic or affective syndrome. ${ }^{10}$ It also provides an argument that vitamin $\mathrm{B}_{12}$ deficiency should be considered in any person with an unusual psychiatric presentation, not only when this is 'organic', and regardless of the absence of macrocytosis or anaemia. This is particularly important since delayed diagnosis can be associated with lack of reversibility.

\section{Sameer Jauhar, MBChB, BSC(Hons), MRCPsych, Department of Psychiatry, \\ Hairmyres Hospital, East Kilbride, UK; Allison Blackett, MA(Hons), DClinPsy, Esteem Glasgow (South), Brand Street Resource Centre, Glasgow, UK; Pavan Srireddy, MBBS, MRCPsych, Department of Psychiatry, Southern General Hospital, Glasgow, UK; Peter J. McKenna, MB, ChB, MRCPsych, Research, Benito Menni Complex Assistencial en Salut Mental, Barcelona, Spain, and Centro de Investigación Biomédica en Red de Salud Mental (CIBERSAM), Spain \\ Correspondence: S. Jauhar, Hairmyres Hospital, East Kilbride, Lanarkshire, G75 8RG, UK. Email: sameerjauhar@yahoo.co.uk \\ First received 12 May 2008, final revision 17 Feb 2010, accepted 28 Feb 2010}

\section{Funding}

P.J.M. is supported by the Instituto de Salud Carlos III, Centro de Investigación en Red de Salud Mental, CIBERSAM.

\section{Acknowledgements}

We thank the patient for giving permission to publish this case report.

\section{References}

1 Lishman WA. Organic Psychiatry: The Psychological Consequences of Cerebral Disorder. Blackwell Science, 1998.

2 Berry N, Sagar R, Tripathi BM. Catatonia and other psychiatric symptoms with vitamin B12 deficiency. Acta Psychiatr Scand 2003; 108: 156-9.

3 Mioshi E, Dawson K, Mitchell J, Arnold R, Hodges JR. The Addenbrooke's Cognitive Examination Revised (ACE-R); a brief cognitive test battery for dementia screening. Int J Geriatr Psychiatry 2006; 21: 1078-85.

4 Wilson B, Cockburn J, Baddeley AD. Rivermead Behavioural Memory Test. Thames Valley Test Company, 1985.

5 Burgess PW, Shallice T. The Hayling and Brixton Tests. Thames Valley Test Company, 1997.

6 Goldberg TE, Goldman RS, Burdick KE, Malhotra AK, Lencz T, Patel RC, et al. Cognitive improvement after treatment with second-generation antipsychotic medications in first-episode schizophrenia: is it a practice effect? Arch Gen Psychiatry 2007; 64: 1115-22.

7 Eastley R, Wilcock GK, Bucks RS. Vitamin B12 deficiency in dementia and cognitive impairment: the effects of treatment on neuropsychological function. Int J Geriatr Psychiatry 2000; 15: 226-33.

8 Vry MS, Haerter K, Kastrup O, Gizewski E, Frings M, Maschke M. Vitamin B12-deficiency causing isolated and partially reversible leukoencephalopathy. J Neurology 2005; 252: 980-2.

9 Lindenbaum J, Healton EB, Savage DG, Brust JC, Garrett TJ, Podell ER. Neuropsychiatric disorders caused by cobalamine deficiency in the absence of anemia or macrocytosis. NEJM 1988; 318: 1720-8.

10 Fink M, Shorter E, Taylor MA. Catatonia is not schizophrenia: Kraepelin's error and the need to recognize catatonia as an independent syndrome in medical nomenclature. Schizophr Bull 2010; 36: 314-20. 